\title{
Seasonal Variations of Melatonin Secretion in Young Females under Natural and Artificial Light Conditions in Fukuoka, Japan
}

\author{
Tomoko Ueno-Towatari ${ }^{1)}$, Kyoko Norimatsu ${ }^{1)}$, Krzysztof Blazejczyk ${ }^{2,3}$, \\ Hiromi Tokura $^{4)}$ and Takeshi Morita ${ }^{1)}$ \\ 1) Department of Living Environmental Science, Fukuoka Women's University \\ 2) Institute of Geography, Kazimierz Wielki University of Bydgoszcz, Poland \\ 3) Institute of Geography and Spatial Organization, Poland \\ 4) Institute of Textile \& Clothing, The Hong Kong Polytechnic University, China
}

\begin{abstract}
The purpose of this study was to examine the seasonal variations of melatonin secretion of subjects and of their surrounding light conditions. Eight Japanese female students $(20.1 \pm 2.6$ yrs, Mean \pm SD) living in Fukuoka, Japan, participated in the present study. Saliva samples were collected every 3 hours over the course of a day, and the light intensity during daily life was measured every 1 min for 5 days in the four seasons. Almost all subjects had different melatonin secretory profiles in autumn, with only two subjects showing similar rhythms in all four seasons. The peak values of melatonin secretion calculated by a spline interpolation were higher in autumn than those in other seasons $(p<0.001$, Fisher's PLSD) and its peak time in this season was significantly delayed compared with those in spring and summer ( $p<0.05$, Fisher's PLSD). The amount of time during daytime exposure to light of $>1,000$ lux was at least thirty minutes in all the seasons, and there were no significant differences among them. The relationship between peak level of melatonin secretion and amount of time of daytime light exposure to $>1,000$ lux was significant only in the autumn. During this season, there was a significant positive correlation $(r=0.83, p<0.05, n=6)$, except for two subjects, whose melatonin secretion remained low. $J$ Physiol Anthropol 26(2): 209-215, 2007 http://www.jstage.jst.go.jp/browse/jpa2
\end{abstract}

[DOI: 10.2114/jpa2.26.209]

Keywords: circadian rhythm, melatonin, light condition, seasonal variation

\section{Introduction}

Melatonin is the primary hormone of the pineal gland, and exhibits a circadian rhythm with the highest levels at night. It is controlled by a pacemaker located in the suprachiasmatic nuclei (SCN) of the hypothalamus (Arendt and Skene, 2005;
Illnerova et al., 2000; Lerchl et al., 1995; Vaughan GM et al., 1978). In mammals, it has been shown that the circadian pacemaker is photoperiod (day-length) dependent (Goldman, 2001; Sumova et al., 1995). Melatonin also exhibits seasonal variations in its circadian rhythm, and such seasonality is well documented in animals and is considered to be a reaction to seasonal changes in day-length (Karsch et al., 1986; Lincoln et al., 2003; Woodfill et al., 1994).

Wehr (1998) demonstrated that the human nocturnal melatonin secretion synchronized with a controlled light-dark cycle in humans, and he also suggested that the duration of nocturnal human melatonin secretion under constant dim light is governed by the duration of the dark period (scotoperiod) to which an individual was most recently chronically exposed, suggesting that seasonal changes in duration of the natural scotoperiod could trigger the seasonal responses of melatonin secretion. Honma et al. (1992) reported that plasma melatonin rhythm showed an earlier peak time in summer (long daylength, strong solar radiation) than in winter (short day-length, weak solar radiation), while Vondrasova et al. (1997) reported that only morning melatonin decline was advanced in the summer compared with the winter. These studies were carried out at a research facility, however, and so the subjects might have been subjected to an unusual experimental time-schedule or unexpected psychosocial stress. Such seasonal variations of melatonin secretion, as a response to a change of day-length, may not apply to modern humans who have less exposure to natural daylight during the daytime and are exposed to artificial light at night.

In this study, we have measured the seasonal rhythms of melatonin secretion and actual light exposure of subjects during their normal daily activities. We have also investigated whether the melatonin secretion rhythm and light exposure displayed seasonality, and how surrounding light conditions were related to the secretory rhythm of melatonin. 


\section{Methods}

\section{Subjects}

Eight healthy female students $(20.1 \pm 2.6$ yrs, Mean \pm SD) participated in the present study. They were Japanese living in Fukuoka Japan (latitude $33^{\circ} 35^{\prime} \mathrm{N}$ ). All subjects were nonsmokers, could sleep well at night, and had a good appetite. They were in good health, were not taking any medication and had no psychological problems.

The experimental procedures were fully explained to each subject before the beginning of the experiment. They were paid for their participation.

\section{Procedures}

This research was carried out in the field four times during the course of a year around the winter solstice, spring equinox, summer solstice and autumn equinox. The experimental period was from Monday to Friday in each of the four seasons. Subjects were required to sleep from 23:00 h until 07:00 h in darkness, and to undertake their regular daily work during the experimental days.

Subjects were instructed to wear continuously an Actiwatch (Mini-Mitter Comp., Inc.) by which the intensity of light exposure and accumulated movement activity levels were recorded every $1 \mathrm{~min}$. Saliva samples were collected every three hours during the course of a day from 10:00 h on Thursday to $07: 00 \mathrm{~h}$ on Friday, which excluded the time of menstruation (because some subjects reported periodic taking of analgesics during that period), using collection tube Salivettes (Sarstedt, Rommelsdorf, Germany), for measurement of melatonin levels. Before the experiment, the subjects were told how to collect saliva samples using Salivettes; they were also instructed not to take caffeine, alcohol, or medicine during the experiment, not to eat, drink, or brush their teeth during the period 90 min before a sampling time, and to rinse their mouth with water $30 \mathrm{~min}$ before the diurnal sampling time. As for nocturnal saliva collection, subjects were asked to set the clock-timer to three hours, and collect saliva in darkness.

Samples were immediately stored at $-5^{\circ} \mathrm{C}$ in a refrigerator and moved to the laboratory the next day. The saliva samples were subsequently stored at $-20^{\circ} \mathrm{C}$ in the laboratory until thawed for melatonin measurement. Salivary melatonin was measured by commercial ELISA kits (Buhlmann Laboratories AG Swiss).

Since melatonin levels were the data obtained every three hours, the spline interpolation, which produced smooth fit curves without changing the original points, was applied to the calculation of peak level and peak time of melatonin secretion.

\section{Statistics}

Statistical analysis was performed by two-way repeated measures and one-way analysis of variance (ANOVA) followed by Fisher's PLSD post hoc tests, and assessment of Pearson's correlation coefficient.

\section{Results}

Mean and individual melatonin rhythms are shown in Figures 1 and 2. Figure 1 shows the group means; there were typical melatonin circadian rhythms, i.e., rising in the evening and declining in the early morning. There were significant differences and interaction between the seasons $(p<0.001$, repeated measures ANOVA). In winter, spring and summer, melatonin rhythms showed a similar tendency, while those levels at 4:00 h and 7:00 h were significantly higher in autumn than in other seasons $(p<0.001$, Fisher's PLSD). Melatonin peak time in autumn also seems to be delayed compared with other seasons. This would account for the interaction effect.

Table 1 shows peak level and peak time of melatonin secretion calculated by spline interpolation. The peak level was significantly higher in autumn compared with other seasons $(p<0.001$, Fisher's PLSD), and the time of the peak was also

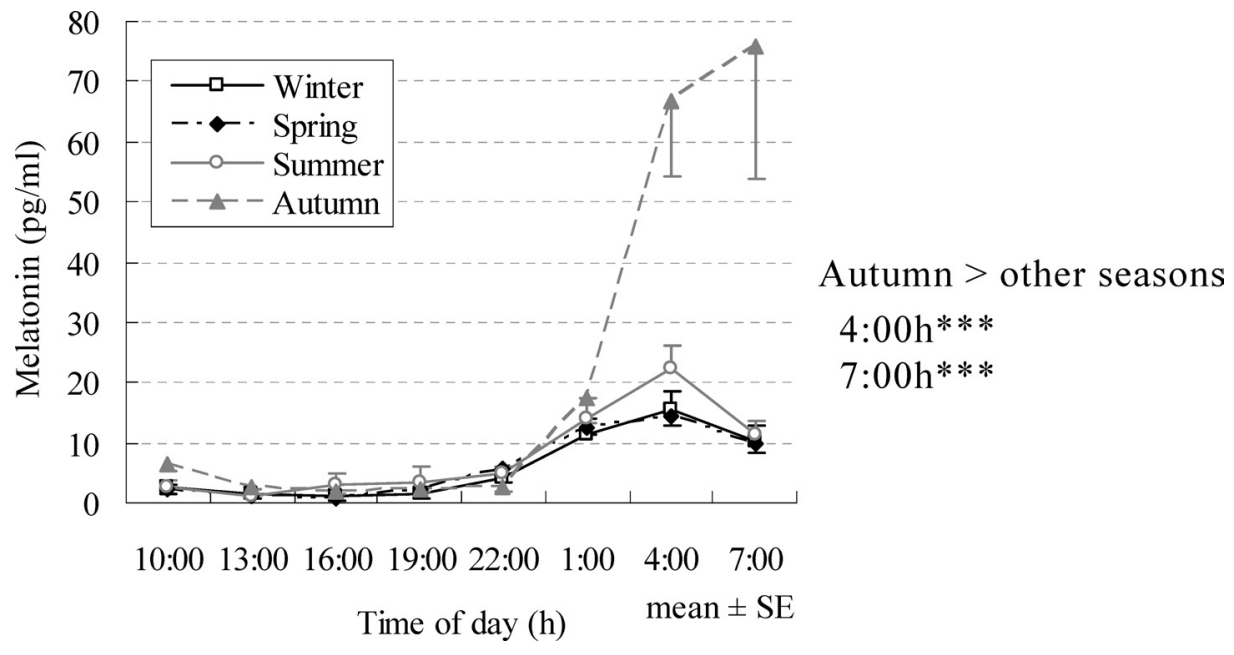

Fig. 1 Seasonal variations of the melatonin rhythm $(n=8)$. Values are means \pm SE. $* * * p<0.001$, Fisher's PLSD. 

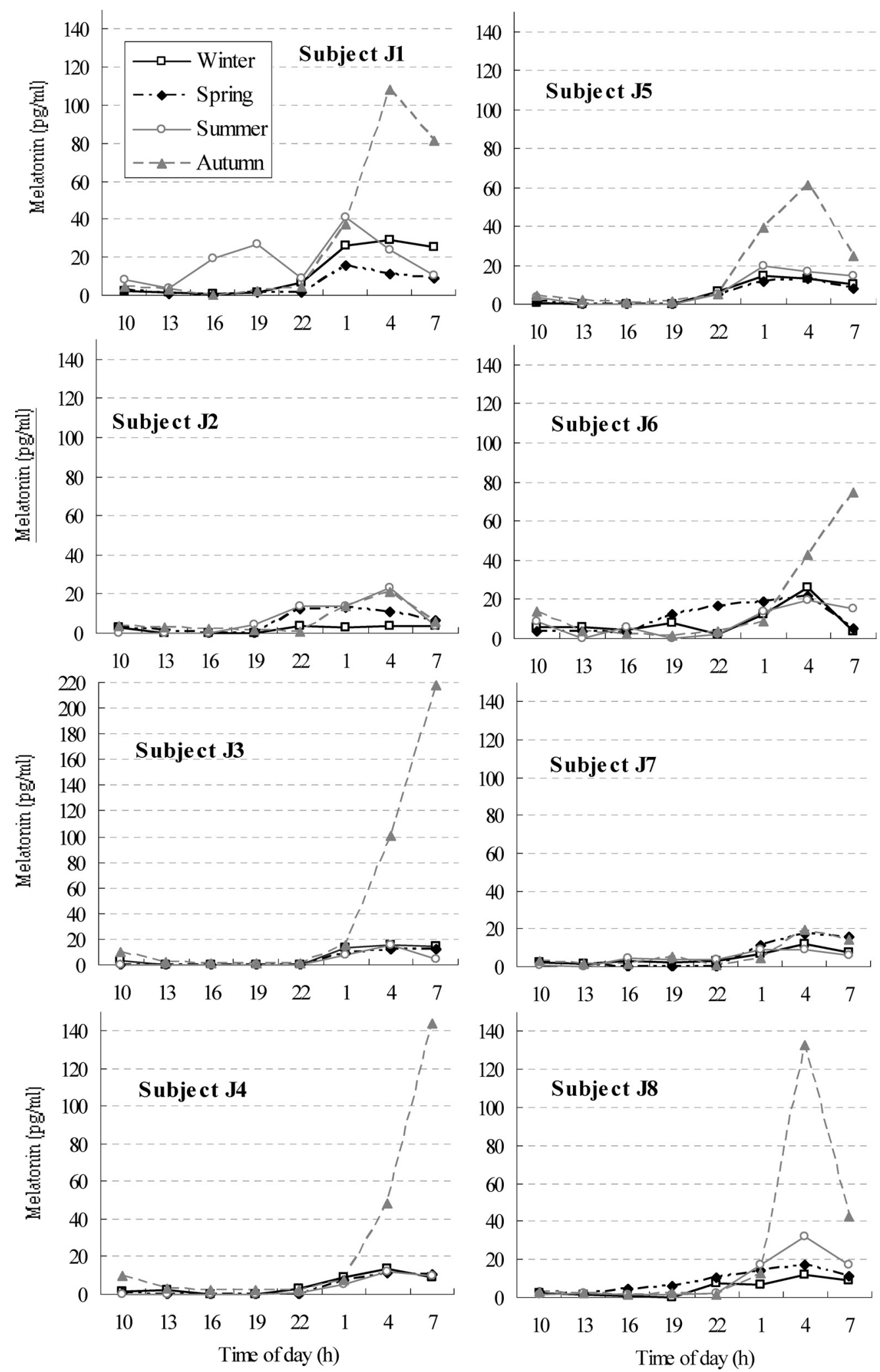

Fig. 2 Seasonal variations of individual melatonin rhythm $(n=8)$. 
significantly delayed in autumn compared with spring and summer $(p<0.05$, Fisher's PLSD). Individuals' melatonin rhythms show these differences, except for subjects J2 and J7 (Figure 2).

Concerning the light intensity and the exposed time to light in the evening which the subjects experienced, those were very small, and there did not exist any seasonal variation. The total time (between 07:00 $\mathrm{h}$ and 16:00 h) which subjects spent under bright light was calculated for each subject and then compared with that individual's melatonin rhythm. Bright light was decided as $>1,000$ lux which was based on the reports (Cole et al., 1995; Espiritu et al., 1994). Mean exposure times were

Table 1 Peak level and peak time of melatonin secretion calculated by spline interpolation.

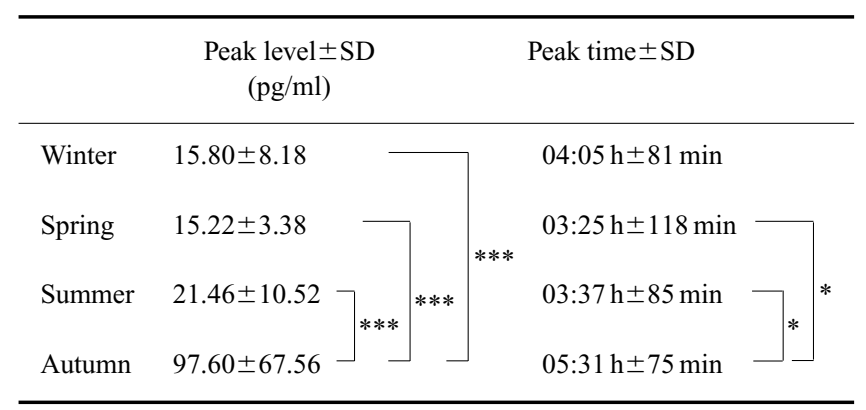

$* * * p<0.001, * p<0.05$, respectively, Fisher's PLSD.
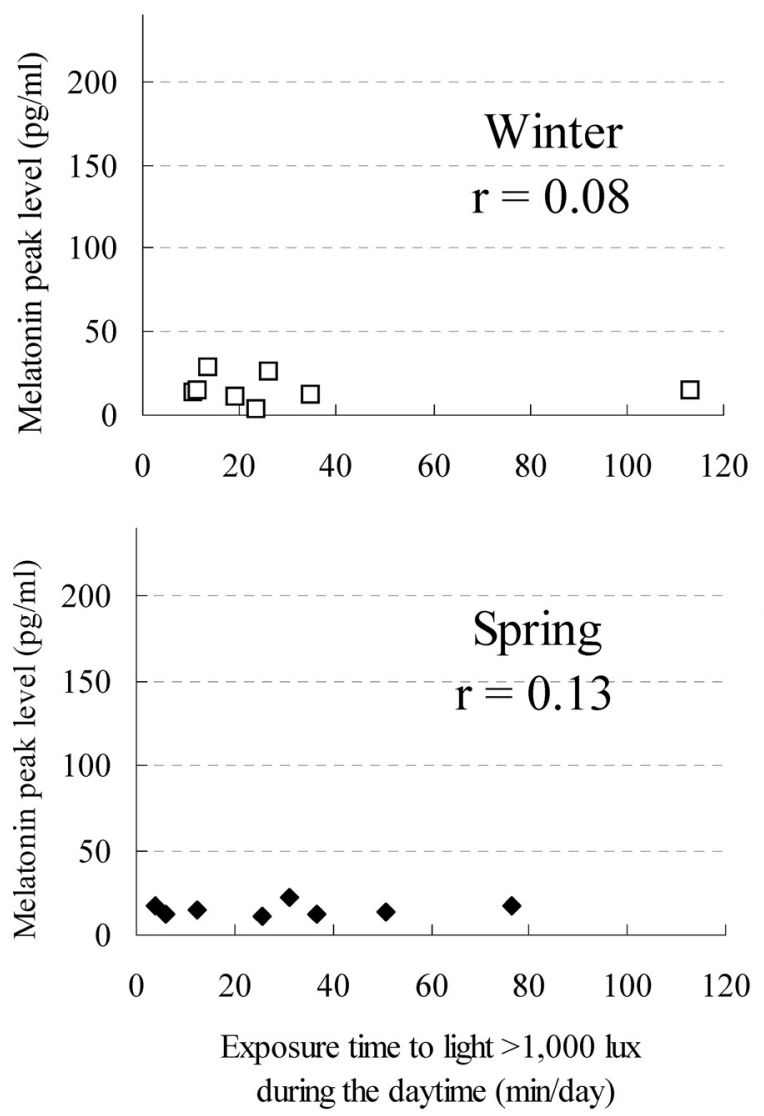

$31.40 \pm 33.97(\mathrm{~min} /$ day $\pm \mathrm{SD})$ in winter, $30.31 \pm 24.53$ in spring, $37.85 \pm 15.59$ in summer and $33.94 \pm 13.25$ in autumn. Mean exposure times were $>30 \mathrm{~min}$ in all four seasons, and there were no significant differences between these times. Figure 3 shows the relationship between the peak level of melatonin secretion and the amount of time of exposure to $>1,000$ lux in each season; none of the correlations was significant. However, the distribution only in autumn seemed to have some remarkable trends. Most subjects showed different characteristics of melatonin peak level and peak time in autumn from those in other seasons, while subjects $\mathrm{J} 2$ and $\mathrm{J} 7$ did not have such behavior in autumn (Fig. 2). We therefore re-analyzed these data excluding subjects $\mathrm{J} 2$ and $\mathrm{J} 7$. In this case, a significant correlation was found between peak level of melatonin secretion and exposure time to light $>1,000$ lux in the autumn $(r=0.83, p<0.05$, Fig. 4 ), but subjects still had light exposure times that did not differ significantly between the seasons.

Activity data during the daytime (between 07:00h and 16:00h) was also calculated in each season and subject. Mean activities were $12.97 \pm 28.34\left(\times 10^{3}\right.$ count \pm SD $)$ in winter, $12.33 \pm 21.85$ in spring, $14.65 \pm 37.35$ in summer, and $13.11 \pm$ 27.31 in autumn. There were no significant differences between the seasons, though there were significant differences between the subjects $(p<0.001$, repeated measures ANOVA). However, we could not find any specific relationships between melatonin behavior and individual's activity.
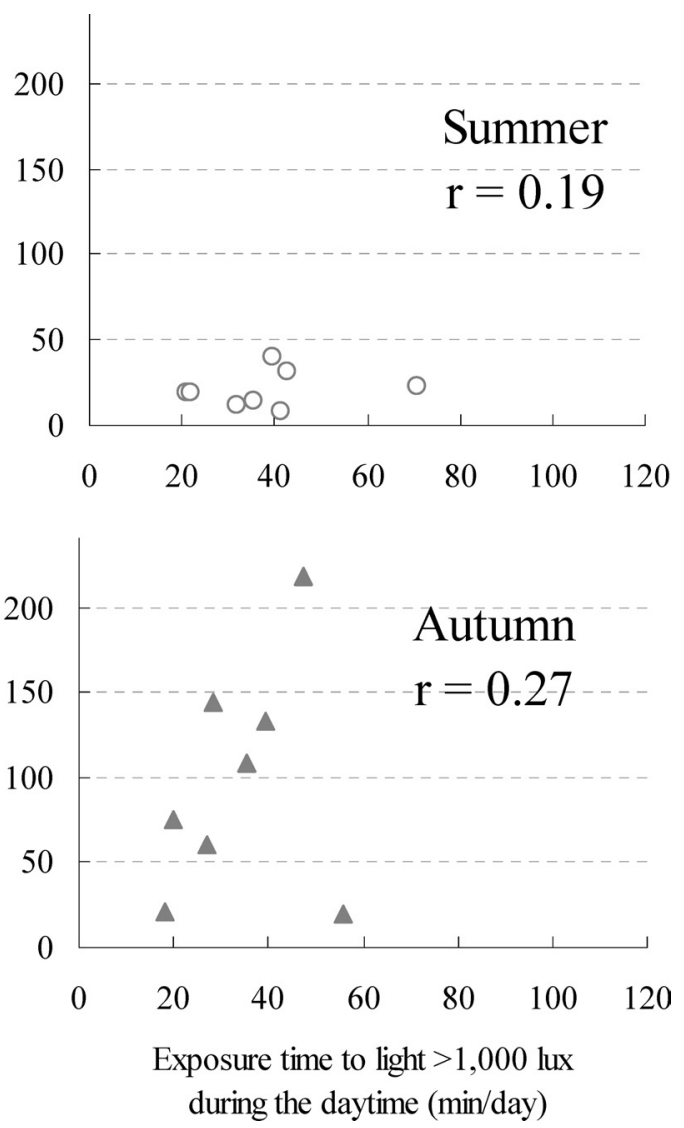

Fig. 3 Relationship between peak level of melatonin secretion and exposure time to light $>1,000$ lux during the daytime (each season, $n=8$ ). 


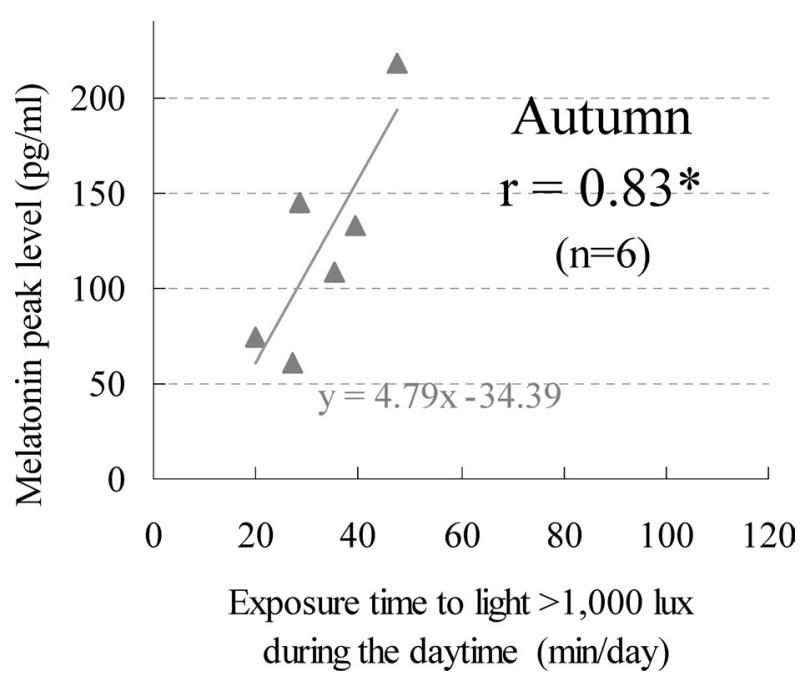

Fig. 4 Relationship between peak level of melatonin secretion and exposure time to light $>1,000$ lux during the daytime (autumn, $n=6$, excluding subjects $\mathrm{J} 2$ and $\mathrm{J} 7$ ).

$* p<0.05$, Pearson's correlation coefficient test.

\section{Discussion}

In autumn, melatonin circadian rhythms differed from the other seasons: peak levels of melatonin secretion were higher, and the peak time of melatonin secretion was delayed. Furthermore, there was a significant correlation between the peak level of melatonin secretion and the amount of time of exposure to daytime light $>1,000$ lux only in the autumn, when the relationship was direct. The present results were inconsistent with previous reports that showed an advance of melatonin peak level or morning decline in summer (Honma et al., 1992; Vondrasova et al., 1997), but no such shift of melatonin secretion was observed in summer in this study. This discrepancy in summer may reflect the modern lifestyle of humans, i.e., they are little exposed to natural daylight during the daytime and exposed to artificial lighting after dusk. In fact, in the current study, mean times that subjects were exposed in the daytime to light $>1,000$ lux were below $40 \mathrm{~min} /$ day, even in the summer, when there is a long photoperiod.

Two questions arise from our results: Why did the melatonin rhythm show a higher peak level and delayed peak time in autumn? Why did subjects J2 and J7 show different behavior during this season?

It has been reported that bright light exposure during the daytime affected plasma and urinary melatonin rhythm in humans (Hashimoto et al., 1997; Park and Tokura, 1999). Hashimoto et al. (1997) showed that midday exposure to bright light advanced the onset phase, but not the end phase, of plasma melatonin. On the other hand, Park and Tokura (1999) showed greater melatonin in urine during the night when subjects received the bright light during the daytime. In our study, subjects were exposed to bright light mainly during the daytime, and the difference of the amount of bright light exposure between the seasons was not statistically significant. However, the melatonin rhythm in autumn exhibited a greater peak level and delayed peak time.

The quality of light may be one of factors causing this seasonality in the melatonin rhythm. It has been reported that exposure of light quality had different effects on melatonin secretion depending on its wavelength. Morita et al. (1995; 1997) found that green light irradiation of $2,500 \mathrm{lux} \times 5 \mathrm{~h}$ during the morning or that of $1,000 \operatorname{lux} \times 5 \mathrm{~h}$ during the evening inhibited nocturnal urinary melatonin levels compared with red light. In the present study, the natural sunlight and/or artificial light the subjects were exposed to in autumn might have had different wavelengths compared with the light during other seasons, and/or subjects $\mathrm{J} 2$ and $\mathrm{J} 7 \mathrm{might}$, because of their lifestyle, have been exposed to different light intensity and wavelengths. It is important to collect more data pertinent to this issue. Such measurements, of the wavelengths of light received by subjects during their daily life, are a topic for a future study.

There are several studies which observed an increase of the human serum melatonin level in the luteal phase compared to the follicular phase during the menstrual cycle (Arendt, 1978; Webley and Leidenberger, 1986). Opposite to this, Ito et al. (1995) and Kivela et al. (1988) reported no significant effect of the menstrual cycle on plasma or serum melatonin levels, but Kivela et al. (1988) also found that the mean nighttime serum melatonin levels were significantly higher in winter than in summer during the follicular phase. This seasonal difference observed in our study might have been caused by higher peak level and/or shifted peak time of melatonin rhythm during the follicular phase in winter compared with in summer, because their sampling time was a 6-hour interval. As mentioned above, it still remains to be discovered whether the menstrual cycle and its seasonality effects correlate to melatonin secretion. Thus, such a menstrual cycle of subjects on a saliva sampling day might have affected seasonal and individual differences of melatonin peak level and peak time in this study.

On the other hand, seasonal variation in the amplitude of the melatonin peak was displayed in animal studies such as the European hamster (Garidou et al., 2003; Vivien-Roels et al., 1992) and the Siberian hamster (Ribelayga et al., 2000). Garidou et al. (2003) reported that the European hamster displayed an annual variation in the nocturnal peak of pineal melatonin content with a small peak (5-fold increase at night; 4-h duration) in a naturally long photoperiod (June) and a large one (15-fold nocturnal increase; 9-h duration) in a naturally short photoperiod (November). Surprisingly, they also reported the amplitude of the nocturnal melatonin peak was significantly lower in indoor hamsters kept for 8 wk under an artificially short photoperiod compared with outdoor hamsters in November. The factors that vary throughout the year (e.g., stress of psychosocial events and mood) might have affected the melatonin rhythm, and ambient temperature and day-length varying with the season might also have triggered the seasonal change of melatonin behavior observed in this study. However, 
the present study does not enable us to conclude which factors might have been responsible for the seasonal rhythm of melatonin. Although a precise answer to the cause could not be fully explained, at least it is a fact that there was a remarkable correlation between the higher melatonin peak level and the amount of time during daytime exposure to light of $>1,000$ lux in autumn. Such a phenomenon might imply a seasonal change in the individual's sensitivity to light, followed by an increase of the melatonin peak level in the autumn in humans. Aizawa et al. (2002) reported that melatonin administration reduced human tympanic temperature $\left(T_{\text {ty }}\right)$ and it made cutaneous vasodilation and local sweating of the forearm occur at significantly lower $T_{\text {ty }}$ during local body warming in a hot water bath. They concluded that melatonin administration lowers the thermoregulatory setpoint.

The higher melatonin peak level in autumn seems to be helpful for cold resistance by reducing the core temperature, and it may be an adaptive mechanism for the onset of the cold winter.

Acknowledgements The authors thank Prof. Dr. Jim Waterhouse, Liverpool, UK, for his kind correction of our English text. This research was supported by the Japan Society for the Promotion of Science Grant No. 16207021.

\section{References}

Aizawa S, Tokura H, Morita T (2002) The administration of exogeneous melatonin during the daytime lowers the thermoregulatory setpoint in humans. J Therm Biol 27: 115-119

Arendt J, Skene DJ (2005) Melatonin as a chronobiotic. Sleep Med Rev 9: 25-39

Arendt J (1978) Melatonin assays in body fluids. J Neural Transm Suppl 13: 265-278

Cole RJ, Kripke DF, Wisbey J, Mason WJ, Gruen W, Hauri PJ, Juarez S (1995) Seasonal variation in human illumination exposure at two different latitudes. J Biol Rhythms 10: 324-334

Espiritu RC, Kripke DF, Ancoli-Israel S, Mowen MA, Mason WJ, Fell RL, Klauber MR, Kaplan OJ (1994) Low illumination experienced by San Diego adults: association with atypical depressive symptoms. Biol Psychiatry 35: 403-407

Garidou ML, Vividen-Rodels B, Pevet P, Miguez J, Simonneaux V (2003) Mechanisms regulating the marked seasonal variation in melatonin synthesis in the European hamster pineal gland. Am J Physiol Regul Integr Comp Physiol 284: R1043-R1052

Goldman BD (2001) Mammalian photoperiodic system: formal properties and neuroendocrine mechanisms of photoperiodic time measurement. J Biol Rhythms 16: 283-301

Hashimoto S, Kohsaka M, Nakamura K, Honma H, Honma S, Honma K (1997) Midday exposure to bright light changes the circadian organization of plasma melatonin rhythm in humans. Neurosci Lett 221: 89-92

Honma K, Honma S, Kohsaka M, Fukuda N (1992) Seasonal variation in the human circadian rhythm: dissociation between sleep and temperature rhythm. Am J Physiol 262: R885-R891

Illnerova H, Sumova A, Travickova Z, Jac M, Jelinkova D (2000) Hormones, subjective night and season of the year. Physiol Res 49: S1-S10

Ito M, Kohsaka M, Honma K, Fukuda N, Honma S, Katsuno Y, Kawai I, Honma H, Morita N, Miyamoto T, Koyama T (1995) Changes in biological rhythm and sleep structure during the menstrual cycle in healthy women. Seishin Shinkeigaku Zasshi 97: 155-164 [in Japanese with English abstract]

Karsch FJ, Bittman EL, Robinson JE, Yellon SM, Wayne NL, Olster DH, Kaynard AH (1986) Melatonin and photorefractoriness: loss of response to the melatonin seasonal reproductive transitions in the ewe. Biol Reprod 34: 265-274

Kivela A, Kauppila A, Ylostalo P, Vakkuri O, Leppaluoto J (1988) Seasonal, menstrual and circadian secretions of melatonin, gonadotropins and prolaction in women. Acta Physiologica Scandinavia 132: 321-327

Lerchl A, Partsch CJ, Nieschlag E (1995) Circadian and ultradian variations of pituitary and pineal hormones in normal men. J Pineal Res 18: 41-48

Lincoln GA, Andersson H, Loudon A (2003) Clock genes in calendar cells as the basis of annual timekeeping in mammals-a unifying hypothesis. J Endocrinol 179: 1-13

Morita T, Teramoto Y, Tokura H (1995) Inhibitory effect of light of different wavelengths on the fall of core temperature during the nighttime. Jpn J Physiol 45: 667-671

Morita T, Tokura H, Wakamura T, Park SJ, Teramoto Y (1997) Effects of the morning irradiation of light with different wavelengths on the behaviour of core temperature and melatonin in humans. Appl Human Sci 16: 103-105

Park SJ, Tokura H (1999) Bright light exposure during the daytime affects circadian rhythms of urinary melatonin and salivary immunoglobulin A. Chronobiol Int 16: 359-371

Ribelayga C, Pevet P, Simonneaux V (2000) HIOMT drives the photoperiodic changes in the amplitude of the melatonin peak of the Siberian hamster. Am J Physiol Regul Integr Comp Physiol 278: R1339-R1345

Sumova A, Travnickova Z, Peters R, Schwartz WJ, Illnerova H (1995) The rat suprachiasmatic nucleus is a clock for all seasons. Proc Natl Acad Sci USA 92: 7754-7758

Vaughan GM, Allen JP, Tullis W, Siler-Khodr TM, de la Pena A, Sackman JW (1978) Overnight plasma profiles of melatonin and certain adenohypophyseal hormones in men. J Clin Endocrinol Metab 47: 566-571

Vivien-Rodels B, Pevet P, Masson-Pevet M, Canguilhem B (1992) Seasonal variations in the daily rhythm of pineal gland and/or circulating melatonin and 5-methoxytryptophol concentrations in the European hamster, Cricetus cricetus. 
Gen Comp Endocrinol 86: 239-247

Vondrasova D, Hajek I, Illnerova H (1997) Exposure to long summer days affects the human melatonin and cortisol rhythms. Brain Res 759: 166-170

Webley GE, Leidenberger F (1986) The circadian pattern of melatonin and its positive relationship with progesterone in women. J Clin Endocrinol Metab 63: 323-328

Wehr TA (1998) Effect of seasonal changes in daylength on human neuroendocrine function. Horm Res 49: 118-124

Woodfill CJ, Wayne NL, Moenter SM, Karsch JF (1994) Photoperiodic synchronization of a circannual reproductive rhythm in sheep: identification of season-specific time cures. Biol Reprod 50: 965-976
This article was presented at the 8th International Congress of Physiological Anthropology, 2006 (ICPA 2006), in Kamakura, Japan.

Received: September 29, 2006

Accepted: December 22, 2006

Correspondence to: Tomoko Ueno-Towatari, Department of Living Environmental Science, Fukuoka Women's University, 1-1-1 Kasumigaoka, Higashi-ku, Fukuoka 813-8529, Japan

Phone: +81-92-661-2411

Fax: +81-92-661-2415

e-mail: ueno@fwu.ac.jp 